ACTA AGROBOTANICA

Vol. 61 (2): 175-187

2008

\title{
MALTING BARLEY SEED HEALTH DEPENDING ON DIFFERENT FUNGICIDE TREATMENT
}

\author{
Barbara Majchrzak $^{1}$, Bogdan Dubis ${ }^{2}$, Adam Okorski ${ }^{3}$
}

\author{
${ }^{1}$ Department of Phytopathology and Entomology UWM \\ Prawocheńskiego 17, 10-722 Olsztyn, Poland \\ ${ }^{2}$ Department of Agrotechnology and Crop Management \\ M. Oczapowskiego 8, 10-719 Olsztyn, Poland \\ ${ }^{3}$ Department of Diagnostics and Plant Pathophysiology \\ Plac Łódzki 6, 10-727 Olsztyn, Poland \\ e-mail: adam.okorski@uwm.edu.pl
}

Received: 20.01.2008

S u m m a r y

Field studies were carried out in the $2004-2005$ growing seasons. The mycological analysis of malting barley (varieties Prestige and Sezam) grains was performed twice: on seeds stored for 30 days and on seeds stored for five months. The influence of fungicide treatment on species diversity and the amount of fungal pathogens on kernels of both varieties of malting barley were determined in the studies. Main fungal pathogens of both varieties of malting barley were field fungal species, such as: Alternaria alternata, Epicocum purpurascens, and fungi of the genus Fusarium. The extension of the grain storage period to five months resulted in an increased share of pathogenic species.

Key words: malting barley, grain, storing, fungi

\section{INTRODUCTION}

In recent years new technologies have been introduced in cereal growing, designed to create optimal conditions for plant growth and development. Adequate protection against diseases in the growing period enables the production of full-value grain suitable for further technological processing and for animal feed purposes. Cereal grains are colonised by many microorganisms, mainly bacteria and fungi. They are initially infected by the so-called "field fungi" which can be saprotrophs or pathogens. Popular field fungi include the following: Alternaria alternata, Epicoccum purpurascens, Fusarium spp. An important pathogenic species often found on barley grains is the fungus Bipolaris sorokiniana, the culprit of cereal seedling and root canker (K n u d s e n et al. 1995). But on stored seeds, the so-called "storage fungi" develop, belonging to the genus Aspergillus, Penicillium or from the family $\mathrm{Mu}-$ coraceae (C h r is t e n s e n, 1972). Grain infection reduces the vigour, restricts germination and is the cause of yield reduction (Š a ri ć et al. 1997). It affects the quality of malt, changes its colour and decreases the taste value. It results from negative changes in the composition of proteins found in the grain. Malting barley grain showing symptoms of strong infection by fungal pathogens cannot be used in the brewing industry; it is only used as animal feed (M athre, 1997). Fungi colonising cereal grains can synthesise numerous secondary metabolites whose presence may be harmful to humans and animals (Munkvold, 2003). Toxic fungal metabolites exhibit cancerogenic, cytogenic, embryogenic, teratogenic and mutagenic effects (P i ę t a , 1997).

The aim of the study was to determine the effect of different intensities of disease protection during the growing period on the health of two varieties of malting barley grain determined after up to 30 days from the harvest and after a 5-month storage period in a floor-storage grain store.

\section{MATERIALS AND METHODS}

The study material was malting barley (Hordeum vulgare L.) grains, varieties Prestige and Sezam, obtained from a strict field experiment carried out at the Agricultural Experimental Station in Bałcyny near the city of Ostróda. 7 methods of barley protection (with different intensity) were used and compared to the untreated control: 


\begin{tabular}{|c|c|c|c|c|c|c|c|c|}
\hline Object & A & B & $\mathrm{C}$ & $\mathrm{D}$ & E & $\mathrm{F}$ & $\mathrm{G}$ & $\mathrm{H}$ \\
\hline & \multicolumn{8}{|c|}{ Dubelt J } \\
\hline $\begin{array}{l}\text { Seed dressing } \\
\text { ВВСН } 00\end{array}$ & - & - & Alert & $\begin{array}{c}\text { Acanto }+ \\
\text { Unix }\end{array}$ & Corbel & $\begin{array}{c}\text { Cerelux } \\
\text { P }\end{array}$ & Corbel & Corbel \\
\hline $\begin{array}{l}\text { 1st treatment } \\
\text { BBCH } 31\end{array}$ & & & & & & & & \\
\hline $\begin{array}{l}\text { 2nd treatment } \\
\text { BBCH } 42\end{array}$ & & & & & Amistar & $\underset{1 / 2}{\text { Amistar }}$ & Amistar & $\begin{array}{c}\text { Alert } \\
+ \\
\text { Cerone }\end{array}$ \\
\hline $\begin{array}{l}\text { 3rd treatment } \\
\text { ВBCH } 72\end{array}$ & - & - & - & - & - & - & Artea & - \\
\hline
\end{tabular}

Doses of preparations were compliant with the recommendations of the Polish Institute of Plant Protection; they were applied at $200 \mathrm{dm}^{-3} \times \mathrm{ha}^{-1}$ of water, following good experimental practice. The active substance included: tebuconazol + triozoxid (Dubelt J), flusilazole and carbendazim (Alert $375 \mathrm{SC}$ ), picoxystrobin (Acanto $250 \mathrm{SC}$ ), fenpropimorph (Corbel 750 EC), flusilazole and fenpropimorph (Cerelux Plus), azoxystrobin (Amistar $250 \mathrm{SC}$ ), propiconazole and cyproconazole (Artea 330 EC), ethephon (Cerone 480 SL). For weed control, the herbicide Mustang $306 \mathrm{SE}$ was used (s.a. - florasulam + 2,4 D).

Malting barley was grown on medium greybrown podzolic soil, belonging to the 3rd agricultural usefulness complex. Sowing was carried out as early as possible, at a spacing of about 370 caryopses per $1 \mathrm{~m}^{2} .45 \mathrm{~kg} \mathrm{P}_{2} 0_{5}, 85 \mathrm{~kg} \mathrm{~K}_{2} \mathrm{O}$ and $40 \mathrm{~kg} \mathrm{~N}$ were applied before sowing. two dates:

Mycological examination was performed on

1) - up to 30 days after the harvest,

2) - five months after the harvest.

The analysis was made using the method designed by N a r k i e w i c z - J o d k o (1991). The grain from the 2004 and 2005 harvest used for analysis was stored in a grain store of the Production and Experimental Facility in Bałcyny, in jute bags.

The pattern of thermal and soil moisture conditions in 2004 was favourable for barley growing. A wet spring guaranteed good water supply for plants at the tillering and shooting stages. But the total rainfall during maturation in July was lower than the long-term mean (Tab. 1). The year 2005 was definitely too dry, and this was the case throughout the whole critical period. The water shortage was alleviated as late as July.

\section{RESULTS}

Following the harvest, 3485 cultures of fungi belonging to 40 species were isolated from the grain of malting barley var. Prestige, in both years of study. The number of isolates in the years of study was different. $21.8 \%$ more fungal cultures were obtained in 2005 than in 2004. But no significant differences were found in the species composition ( 29 and 28 species in the respective years). The species Alternaria alternate was isolated in the greatest number, accounting for $45.88 \%$ of the total colony (Tab. 2). Other fungi colonising the grain in a great number included Epicoccum purpurascens $(10.78 \%)$, Mucor hiemalis $(5.67 \%)$ as well as fungi of the genus Fusarium (30.38\%), being typical pathogens. Among fungi of the genus Fusarium, F. equiseti (544 col.), F. oxysporum (183 col.) and F. poae (93 col.) were predominant. Among other pathogenic fungi, the species Bipolaris sorokiniana (130 col.$3.73 \%$ ) accounted for a large share of the total number of isolates. Botrytis cinerea was isolated sporadically.

Barley grains in the combination $\mathrm{E}$, in which two foliar fungicide treatments were applied, were infected most slightly by fungi. Fungi potentially pathogenic to cereals: + Bipolaris sorokiniana, Botrytis cinerea, Cylindrocarpon destructans and representatives of the genus Fusarium, accounted for 28.8\% (2004) and $31.3 \%$ (2005) in this combination.

After a 5-month storage period, an increase in the number of isolated fungi was observed (Tab. 3). 3679 colonies were obtained, belonging to 36 species. Among these fungi, the species Alternaria alternata was isolated most frequently, accounting for $43 \%$ of the total number of isolates. Cylindrocarpon destructans was also isolated in a great number $(9.8 \%)$. The ge- 
Table 1

Pattern of weather conditions in growing periods in years 2004-2005 (data according to the Meteorological Station in Tomaszkowo).

\begin{tabular}{ccccccc}
\hline Study period & march & april & may & june & july & august \\
\hline \multicolumn{7}{c}{ Daily mean temperature $\left({ }^{\circ} \mathrm{C}\right)$} \\
\hline 2004 & 3 & 8.9 & 11.8 & 15.3 & 17 & 19.2 \\
2005 & -3.1 & 8.2 & 11.6 & 14.2 & 19.7 & 16.9 \\
\hline Long-term mean (1991-2000) & 1.2 & 6.6 & 12.4 & 15.7 & 16.9 & 16.5 \\
\hline & Suma opadów (mm) - Precipitation total (mm) & & \\
\hline 2004 & 28.2 & 51.5 & 87.1 & 90.6 & 78.8 & 41.9 \\
\hline Long-term mean (1991-2000) & 29.3 & 22 & 68.2 & 35.4 & 83.9 & 39.6 \\
\hline
\end{tabular}

nus Fusarium was represented by 13 species, among which $F$. graminearum, $F$. equiseti and $F$. oxysporum dominated. After the 5-month storage period, a slight increase in the number of Epicoccum purpurascens and a marked decline in the number of fungi of the family Mucoraceae, included in typical storage fungi, were observed.

Grains from the control combination were most strongly infected by fungi after the two-month storage period, and grains from the combination $\mathrm{D}$ and $\mathrm{G}$, in which foliar fungicide treatment was applied, were infected in the slightest degree. However, in both these trial objects pathogenic species: Bipolaris sorokiniana, Botrytis cinerea, Cylindrocarpon destructans, and fungi of the genus Fusarium accounted for over $50 \%$ of all isolates.

3260 cultures of fungi belonging to 44 species were isolated from the grain of malting barley var. Sezam (Tab. 4) after the harvest. The so-called "field fungi", belonging to the following species: Alternaria alternata (45.0\%), Fusarium spp. (29.7\%), Epicoccum purpurascens $(10.2 \%)$ and Bipolaris sorokiniana $(6.5 \%)$, were isolated in the greatest number. Typical "storage fungi" accounted for $5.0 \%$, and they were represented by the genera Mucor and Rhizopus. Among fungi of the genus Fusarium, the species F. equiseti and $\mathrm{F}$. poae were predominant.

The smallest number of fungi was isolated from barley grains obtained from the control (untreated) and the combination $\mathrm{G}$ with full chemical protection in the form of seed dressing and three foliar treatments with the fungicides Corbel, Amistar and Artea. The smallest percentage share of pathogenic fungi was noted on barley grains obtained from the trial object B (only seed dressing) and in the first year of study from grains obtained from the combination $\mathrm{G}$.

Potentially pathogenic fungi were represented most numerously in these combinations by Fusarium equiseti and Bipolaris sorokiniana. The species Alternaria alternata and Epicoccum purpurascens dominated in all the combinations.

Out of the storage fungi, Mucor hiemalis as well as single cultures of Mucor circinelloides and Mucor racemosus were isolated quite frequently.

After the 5-month period of storage of barley grain, the number of fungi colonising it did not change significantly, but the percentage shares of particular species changed. A total of 3232 fungal cultures were isolated (33 species) (Tab. 5). A marked increase, by as much as $10 \%$, was found in the number of fungi of the genus Fusarium (from 29.7\% up to 39.1\%). They were represented by 15 species, among which Fusarium equiseti, $F$. avenaceum, $F$. oxysporum were predominant. The number of Epicoccum purpurascens cultures increased. But a significant decline in the number of Alternaria alternata colonies (from $45.0 \%$ down to $36.8 \%$ ) and of fungi of the family Mucoraceae was noted; Bipolaris sorokiniana was also isolated less frequently.

Barley grains from the trial object $\mathrm{B}$, in which only seed dressing was applied, were colonised by fungi in the greatest number. 474 fungal cultures from 21 species were isolated. Among them, pathogenic fungi accounted for $42 \%$ in 2004 and $20 \%$ in 2005 , mainly of the genus Fusarium and Bipolaris sorokiniana. The least number of fungal cultures was isolated from grains obtained from the plots on which full threefold protection was applied (trial object G). 344 cultures belonging to 15 species were obtained. Among them, fungi potentially pathogenic to cereals dominated, in particular from the genus Fusarium, and they accounted for $50 \%$ in 2004 and up to $85 \%$ in 2005 . A similar increase in the number of pathogenic fungi was observed in the trial object $\mathrm{E}$ where the fungicides Corbel and Amistar were applied. 
Table 2

Fungi isolated from grains of spring barley, variety Prestige, 30 days after harvest.

\begin{tabular}{|c|c|c|c|c|c|c|c|c|c|c|c|c|c|c|c|c|c|}
\hline \multirow{3}{*}{ Fungal } & \multicolumn{17}{|c|}{30 days after harvest } \\
\hline & \multicolumn{2}{|c|}{$\mathbf{A}$} & \multicolumn{2}{|c|}{ B } & \multicolumn{2}{|c|}{$\mathbf{C}$} & \multicolumn{2}{|c|}{ D } & \multicolumn{2}{|c|}{$\mathbf{E}$} & \multicolumn{2}{|c|}{$\mathbf{F}$} & \multicolumn{2}{|c|}{ G } & \multicolumn{2}{|c|}{$\mathbf{H}$} & \multirow{2}{*}{$\begin{array}{l}\text { Suma } \\
\text { Total }\end{array}$} \\
\hline & 2004 & 2005 & 2004 & 2005 & 2004 & 2005 & 2004 & 2005 & 2004 & 2005 & 2004 & 2005 & 2004 & 2005 & 2004 & 2005 & \\
\hline \multicolumn{18}{|l|}{ Acremoniella atra } \\
\hline (Corda) Sacc. & 4 & & 2 & & & & & & 2 & & & & & & & & 8 \\
\hline \multicolumn{18}{|l|}{ Acremonium strictum } \\
\hline W. Gams & & & & & 3 & & & & & & & & & & & & 3 \\
\hline \multicolumn{18}{|l|}{ Alternaria alternata } \\
\hline (Fr.) Keissl. & 55 & 98 & 85 & 142 & 103 & 132 & 92 & 112 & 64 & 106 & 98 & 106 & 91 & 112 & 85 & 118 & 1599 \\
\hline \multicolumn{18}{|l|}{ Arthrinium } \\
\hline \multicolumn{18}{|l|}{ phaeospermum (Corda) } \\
\hline M.B. Ellis & & & 3 & & & & & & 2 & & & & & & & & 5 \\
\hline \multicolumn{18}{|l|}{ Arthrinium } \\
\hline \multicolumn{18}{|l|}{ sphaerospermum } \\
\hline Fuckel & & 2 & & 4 & & & & & & & 1 & & & & & 2 & 9 \\
\hline Aspergillus niger Tiegh. & & & & 2 & & & & & & & & 2 & & 2 & & & 6 \\
\hline Aspergillus sp. & & & & & & & & & & & & & & & 1 & & 1 \\
\hline \multicolumn{18}{|l|}{ Aureobasidium bolleyi } \\
\hline (R. Sprague) Arx & & & & & & & 1 & 4 & & & & & 2 & 2 & & & 9 \\
\hline Bipolaris sorokiniana & & & & & & & & & & & & & & & & & \\
\hline (Sacc.) Shoemaker & 18 & 6 & 8 & 10 & 10 & 6 & 6 & 6 & 14 & 2 & 13 & 4 & 5 & 12 & 8 & 2 & 130 \\
\hline Botrytis cinerea Pers. & & & & & & & & 4 & & & & & & & 4 & & 8 \\
\hline Chaetomium globosum & & & & & & & & & & & & & & & & & \\
\hline Kunze & 5 & 2 & & & & 2 & & & & & & & & & & & 9 \\
\hline $\begin{array}{l}\text { Cladosporium } \\
\text { cladosporioides }\end{array}$ & & & & & & & & & & & & & & & & & \\
\hline (Fresen.) G.A. de Vries & & 8 & & & & & & & & & & & 1 & & & & 9 \\
\hline Cladosporium & & & & & & & & & & & & & & & & & \\
\hline herbarum (Pers.) Link & & 2 & & & & & & & & & & & & & & & 2 \\
\hline Cladosporium & & & & & & & & & & & & & & & & & \\
\hline macrocarpum Preuss & 1 & & & & & & & & & & & & & & & & 1 \\
\hline Epicoccum & & & & & & & & & & & & & & & & & \\
\hline purpurascens Ehrenb. & 41 & 14 & 27 & 2 & 18 & 4 & 59 & 16 & 26 & & 30 & 6 & 38 & 26 & 59 & 10 & 376 \\
\hline Fusarium avenaceum & & & & & & & & & & & & & & & & & \\
\hline (Fr.) Sacc. & 5 & & 13 & & 18 & & 4 & 2 & & & & & 5 & & 9 & 2 & 58 \\
\hline Fusarium & & & & & & & & & & & & & & & & & \\
\hline chlamydosporum & & & & & & & & & & & & & & & & & \\
\hline Wollenw. \& Reinking & 2 & & & & & 2 & & & & & & & 1 & & 1 & & 6 \\
\hline Fusarium culmorum & & & & & & & & & & & & & & & & & \\
\hline (W. G. Sm.) Sacc. & & 2 & 1 & 2 & & & 28 & & & & 3 & & 1 & & 41 & & 78 \\
\hline Fusarium equiseti & & & & & & & & & & & & & & & & & \\
\hline (Corda) Sacc. & 8 & 48 & 2 & 96 & & 62 & 2 & 68 & 1 & 58 & 1 & 68 & 6 & 58 & 2 & 64 & 544 \\
\hline Fusarium graminearum & & & & & & & & & & & & & & & & & \\
\hline Schwabe & & & & & & & 1 & & & & & & & & & & 1 \\
\hline
\end{tabular}


cd. table 2

\begin{tabular}{|c|c|c|c|c|c|c|c|c|c|c|c|c|c|c|c|c|c|}
\hline \multicolumn{18}{|l|}{ Fusarium oxysporum } \\
\hline Schltdl. & 35 & & 31 & & 60 & & 3 & & 22 & & & & 30 & & 2 & & 183 \\
\hline \multicolumn{18}{|l|}{ Fusarium poae (Peck) } \\
\hline Wollenw. & & & 2 & 2 & 2 & 2 & & & 1 & & 76 & 2 & 3 & & 3 & & 93 \\
\hline \multicolumn{18}{|l|}{ Fusarium solani (Mart.) } \\
\hline Sacc. & & & & & & & 2 & & & & & & & & & & 2 \\
\hline \multicolumn{18}{|l|}{ Fusarium } \\
\hline sporotrichioides Sherb. & & & & 22 & & & & & & 4 & & 8 & & 4 & & 8 & 46 \\
\hline \multicolumn{18}{|l|}{ Fusarium tricinctum } \\
\hline (Corda) Sacc. & & & & & 2 & & 5 & & & & 1 & & 1 & & 1 & & 10 \\
\hline Fusarium spp. & & & 9 & & & 2 & 3 & 2 & 2 & & 2 & & 4 & & 1 & & 25 \\
\hline \multicolumn{18}{|l|}{ Gliomastix murrorum } \\
\hline (Corda) S. Hughes & & & & & & 2 & & & & & & 2 & & 2 & & & 6 \\
\hline \multicolumn{18}{|l|}{ Gonatobotrys simplex } \\
\hline Corda & & & & & & & & & 1 & & & & 4 & & & & 5 \\
\hline Graphium spp. & & & & & & & & & & & & & & 2 & & & 2 \\
\hline \multicolumn{18}{|l|}{ Humicola fuscoatra } \\
\hline Traaen & & & & & & & & & & & & & & 2 & & & 2 \\
\hline \multicolumn{18}{|l|}{ Microdochium nivale } \\
\hline Hallett & 1 & & 1 & & 4 & & 4 & & & & & & & & 3 & & 13 \\
\hline \multicolumn{18}{|l|}{$\begin{array}{l}\text { Monodyctis glauca } \\
\text { (Cooke et Harkn.) }\end{array}$} \\
\hline Hughes. & & & & & & & & & 3 & & & & & & & & 3 \\
\hline \multicolumn{18}{|l|}{ Monodyctis levis } \\
\hline (Wiltsh.) Hughes. & & & & & & & & & & & & & & & & 2 & 2 \\
\hline \multicolumn{18}{|l|}{ Mucor hiemalis } \\
\hline Wehmer & & 22 & & & & 40 & & 6 & & 34 & & 24 & & 46 & & 24 & 196 \\
\hline Penicillium $s p$ & & & & & & & & & 1 & & & & & & & & 1 \\
\hline \multicolumn{18}{|l|}{ Periconia } \\
\hline \& Aar.G. Johnson & & & & & 3 & & 1 & & & & & & & & & & 4 \\
\hline \multicolumn{18}{|l|}{ Rhizopus nigricans } \\
\hline Ehrenb. & & & 1 & & & 2 & & & & & & & 1 & & & 4 & 8 \\
\hline \multicolumn{18}{|l|}{ Scopulariopsis } \\
\hline Bainier & & 2 & & 2 & & & & 2 & & & & & & & & & 6 \\
\hline \multicolumn{18}{|l|}{ Trichoderma hamatum } \\
\hline non sporulating fungi & & 4 & & & & & & 2 & & & & & & 2 & & & 8 \\
\hline number of isolates & 175 & 212 & 185 & 286 & 223 & 256 & 211 & 228 & 139 & 204 & 225 & 222 & 193 & 270 & 220 & 236 & \\
\hline number of isolates & & & & & & & & & & & & & & & & & 3485 \\
\hline from trials & & & & & & & & 39 & & 43 & & 47 & & 63 & & 56 & \\
\hline
\end{tabular}


Table 3

Fungi isolated from grains of winter barley, variety Prestige, 5 months after harvest.

\begin{tabular}{|c|c|c|c|c|c|c|c|c|c|c|c|c|c|c|c|c|c|}
\hline \multirow{3}{*}{ Fungal } & \multicolumn{17}{|c|}{5 month after harvest } \\
\hline & \multicolumn{2}{|c|}{$\mathbf{A}$} & \multicolumn{2}{|c|}{ B } & \multicolumn{2}{|c|}{ C } & \multicolumn{2}{|c|}{ D } & \multicolumn{2}{|c|}{$\mathbf{E}$} & \multicolumn{2}{|c|}{$\mathbf{F}$} & \multicolumn{2}{|c|}{ G } & \multicolumn{2}{|c|}{$\mathbf{H}$} & \multirow{2}{*}{$\begin{array}{r}\text { Suma } \\
\text { Total }\end{array}$} \\
\hline & 2004 & 2005 & 2004 & 2005 & 2004 & 2005 & 2004 & 2005 & 2004 & 2005 & 2004 & 2005 & 2004 & 2005 & 2004 & 2005 & \\
\hline $\begin{array}{l}\text { Acremoniella atra } \\
\text { (Corda) Sacc. }\end{array}$ & 1 & & 3 & & & & & & & & & & & & & & 4 \\
\hline $\begin{array}{l}\text { Acremonium roseolum } \\
\text { (G. Sm.) W. Gams }\end{array}$ & 2 & & & & & & & & & & & & & & & & 2 \\
\hline $\begin{array}{l}\text { Acremonium strictum } \\
\text { W. Gams }\end{array}$ & 3 & & 1 & & & & & & 3 & & & & & & & & 7 \\
\hline $\begin{array}{l}\text { Alternaria alternata } \\
\text { (Fr.) Keissl. }\end{array}$ & 117 & 125 & 93 & 133 & 77 & 130 & 70 & 109 & 94 & 112 & 24 & 124 & 69 & 114 & 91 & 104 & 1586 \\
\hline $\begin{array}{l}\text { Arthrinium } \\
\text { sphaerospermum Fuckel }\end{array}$ & & & & & & & & 1 & & & & & 2 & & & & 3 \\
\hline $\begin{array}{l}\text { Aureobasidium bolleyi } \\
\text { (R. Sprague) Arx }\end{array}$ & & & & & & & & & & & 5 & & & & 1 & & 6 \\
\hline $\begin{array}{l}\text { Bipolaris sorokiniana } \\
\text { (Sacc.) Shoemaker }\end{array}$ & 12 & 7 & 10 & 12 & 11 & 4 & 8 & 2 & 13 & 10 & 2 & 6 & 8 & 5 & 4 & 4 & 118 \\
\hline Botrytis cinerea Pers. & 1 & 1 & 4 & & & & & & & & & & & & & & 6 \\
\hline $\begin{array}{l}\text { Cladosporium } \\
\text { cladosporioides } \\
\text { (Fresen.) G. A. de Vries }\end{array}$ & 2 & & 1 & & 14 & & 2 & & 6 & & 5 & & 18 & & 7 & & 55 \\
\hline $\begin{array}{l}\text { Cylindrocarpon } \\
\text { destructans (Zinssm.) } \\
\text { Scholten }\end{array}$ & 65 & & 71 & & 65 & & 13 & & 59 & & 20 & & 18 & & 51 & & 362 \\
\hline $\begin{array}{l}\text { Epicoccum purpurascens } \\
\text { Ehrenb. }\end{array}$ & 1 & 50 & 0 & 35 & 6 & 35 & 56 & 46 & 2 & 41 & 1 & 31 & 33 & 46 & 9 & 38 & 430 \\
\hline $\begin{array}{l}\text { Fusarium avenaceum } \\
\text { (Fr.) Sacc. }\end{array}$ & & & 1 & & & & 2 & 1 & 3 & & 3 & 5 & 5 & & & & 20 \\
\hline $\begin{array}{l}\text { Fusarium cerealis } \\
\text { (Cooke) Sacc. }\end{array}$ & & & & & 2 & & & & & & 1 & & & & & & 3 \\
\hline $\begin{array}{l}\text { Fusarium concolor } \\
\text { Reinking }\end{array}$ & & & 3 & & 2 & & 1 & & 1 & & 3 & & & & & & 10 \\
\hline $\begin{array}{l}\text { Fusarium culmorum } \\
\text { (W. G. Sm.) Sacc. }\end{array}$ & 9 & & 2 & & 11 & 6 & 5 & & 4 & & 13 & & 6 & 2 & 3 & & 61 \\
\hline $\begin{array}{l}\text { Fusarium equiseti } \\
\text { (Corda) Sacc. }\end{array}$ & & 16 & & 11 & & 7 & & 28 & & 28 & & 29 & & 8 & & 26 & 153 \\
\hline $\begin{array}{l}\text { Fusarium fusarioides } \\
\text { (Gonz. Frag. } \\
\text { \& Cif.) C. Booth }\end{array}$ & 3 & & 2 & 7 & & 1 & 1 & & & & 2 & 7 & & & & & 23 \\
\hline $\begin{array}{l}\text { Fusarium graminearum } \\
\text { Schwabe }\end{array}$ & 102 & & 62 & & 82 & & 3 & & 63 & & 49 & & 3 & & 61 & & 425 \\
\hline $\begin{array}{l}\text { Fusarium oxysporum } \\
\text { Schltdl. }\end{array}$ & 1 & & 4 & & 4 & & 7 & & 8 & & 45 & & 20 & 2 & 5 & 1 & 97 \\
\hline
\end{tabular}


cd. table 3

\begin{tabular}{|c|c|c|c|c|c|c|c|c|c|c|c|c|c|c|c|c|c|}
\hline $\begin{array}{l}\text { Fusarium poae } \\
\text { (Peck) Wollenw. }\end{array}$ & & & & & & 1 & & & & 1 & & 1 & & 5 & 3 & & 11 \\
\hline \multicolumn{18}{|l|}{ Fusarium } \\
\hline sporotrichioides Sherb. & & & & & & & 2 & & & & 1 & & & & & & 3 \\
\hline Fusarium spp. & 2 & & 3 & & 1 & & 4 & 1 & 4 & & 26 & & 3 & & 9 & & 53 \\
\hline \multicolumn{17}{|l|}{ Fusarium tricinctum } & 122 \\
\hline \multicolumn{18}{|l|}{$\begin{array}{l}\text { Gelasinospora } \\
\text { reticulispora (Greis et } \\
\text { Greis Dengler) }\end{array}$} \\
\hline C. et M. Moreau & & & & & & & & 1 & & & & & & 1 & & & 2 \\
\hline \multicolumn{18}{|l|}{ Gonatobotrys simplex } \\
\hline Corda & & & 2 & & & & & & & & & & & & & & 2 \\
\hline \multicolumn{18}{|l|}{ Humicola fuscoatra } \\
\hline Traaen & & & & & & & & & & & 1 & & & & & & 1 \\
\hline Humicola grisea Traaen & 1 & & & & & & & & & & & & & & & & 1 \\
\hline \multicolumn{18}{|l|}{$\begin{array}{l}\text { Microdochium nivale } \\
\text { (Fr.) Samuels \& I. C. }\end{array}$} \\
\hline Hallett & 4 & & 5 & & 7 & & 11 & & 8 & & 1 & & 7 & 1 & 4 & & 48 \\
\hline \multicolumn{18}{|l|}{$\begin{array}{l}\text { Minimedusa polyspora } \\
\text { (J. W. Hotson) Weresub }\end{array}$} \\
\hline \& P. M. LeClair & & & & & 1 & & & & & & & & & & & & 1 \\
\hline \multicolumn{18}{|l|}{$\begin{array}{l}\text { Monodyctis glauca } \\
\text { (Cooke et Harkn.) }\end{array}$} \\
\hline Hughes. & & & & & & & & & & & & & 1 & & & & 1 \\
\hline \multicolumn{18}{|l|}{ Mortierella alpina } \\
\hline Peyronel & 4 & & 7 & & & & & & & & & & & & & & 11 \\
\hline Mortierella spp. & 4 & & 5 & & 10 & & & & & & & & & & & & 19 \\
\hline Mucor hiemalis Wehmer & & & & & & & & & & & & & 2 & & & & 2 \\
\hline Mucor spp. & & & & & & & & & 2 & & & & & & & & 2 \\
\hline \multicolumn{18}{|l|}{ Periconia macrospinosa } \\
\hline \multicolumn{18}{|l|}{ Lefebvre \& Aar. } \\
\hline G. Johnson & & & & & & & & & & & 1 & & & & & & 1 \\
\hline \multicolumn{18}{|l|}{ Rhizopus nigricans } \\
\hline Ehrenb. & & & 2 & & & & 2 & & 2 & & & & & 4 & 6 & 7 & 23 \\
\hline non sporulating fungi & & & & 1 & 1 & & & & & & & & 1 & & 2 & & 5 \\
\hline number of isolates & 335 & 206 & 283 & 209 & 295 & 213 & 189 & 203 & 272 & 199 & 206 & 221 & 198 & 194 & 263 & 193 & \\
\hline $\begin{array}{l}\text { number of isolates } \\
\text { from trials }\end{array}$ & 54 & & 4 & & 5 & & 3 & & 47 & & 42 & 27 & 39 & 2 & 45 & & $36 / 9$ \\
\hline
\end{tabular}


Table 4

Fungi isolated from grains of winter barley, variety Sezam, 30 days after harvest.

\begin{tabular}{|c|c|c|c|c|c|c|c|c|c|c|c|c|c|c|c|c|c|}
\hline \multirow{3}{*}{ Fungal species } & \multicolumn{17}{|c|}{30 days after harvest } \\
\hline & \multicolumn{2}{|c|}{ A } & \multicolumn{2}{|c|}{ B } & \multicolumn{2}{|c|}{ C } & \multicolumn{2}{|c|}{$\mathrm{D}$} & \multicolumn{2}{|c|}{$\mathrm{E}$} & \multicolumn{2}{|c|}{$\mathrm{F}$} & \multicolumn{2}{|c|}{ G } & \multicolumn{2}{|c|}{$\mathrm{H}$} & \multirow{2}{*}{$\begin{array}{r}\text { Suma } \\
\text { Total }\end{array}$} \\
\hline & 2004 & 2005 & 2004 & 2005 & 2004 & 2005 & 2004 & 2005 & 2004 & 2005 & 2004 & 2005 & 2004 & 2005 & 2004 & 2005 & \\
\hline Acremoniella atra & & & & & & & & & & & & & & & & & \\
\hline (Corda) Sacc. & & & & & & & & & 1 & & & & & & & & 1 \\
\hline Acremonium strictum & & & & & & & & & & & & & & & & & \\
\hline W. Gams & & & 5 & & & & & & & & & & & & & & 5 \\
\hline $\begin{array}{l}\text { Alternaria alternata } \\
\text { (Fr.) Keissl. }\end{array}$ & 81 & 66 & 121 & 110 & 104 & 84 & 138 & 92 & 51 & 88 & 79 & 108 & 91 & 80 & 77 & 100 & 1470 \\
\hline $\begin{array}{l}\text { Arthrinium sphaero- } \\
\text { spermum Fuckel }\end{array}$ & & & & & & 4 & & & 1 & & & & & & & & 5 \\
\hline $\begin{array}{l}\text { Aureobasidium bolleyi } \\
\text { (R. Sprague) Arx }\end{array}$ & & 2 & 3 & 4 & & 4 & & 2 & & 2 & & & & & & 2 & 19 \\
\hline $\begin{array}{l}\text { Aureobasidium pul- } \\
\text { lulans (de Bary) G. } \\
\text { Arnaud }\end{array}$ & & & & & & & & & 2 & & & & & & & & 2 \\
\hline $\begin{array}{l}\text { Bipolaris sorokiniana } \\
\text { (Sacc.) Shoemaker }\end{array}$ & 18 & 12 & 5 & 10 & 23 & 18 & 7 & 14 & 26 & 8 & 15 & 10 & 9 & 30 & 2 & 4 & 211 \\
\hline Botrytis cinerea Pers. & & & 1 & & & & & & & & & 2 & & & & & 3 \\
\hline $\begin{array}{l}\text { Chaetomium globosum } \\
\text { Kunze }\end{array}$ & & & 1 & & & & & & 4 & & & & & & & & 5 \\
\hline $\begin{array}{l}\text { Cladosporium clado- } \\
\text { sporioides (Fresen.) } \\
\text { G. A. de Vries }\end{array}$ & & 2 & 3 & 2 & & & & & & 2 & & 2 & & & & 2 & 13 \\
\hline $\begin{array}{l}\text { Cladosporium } \\
\text { macrocarpum Preuss }\end{array}$ & & & 5 & & & & & & & & & & & & & & 5 \\
\hline $\begin{array}{l}\text { Epicoccum } \\
\text { purpurascens Ehrenb. }\end{array}$ & 34 & 10 & 26 & 12 & 48 & & 22 & 14 & 42 & 8 & & & 68 & & 41 & 8 & 333 \\
\hline $\begin{array}{l}\text { Fusarium avenaceum } \\
\text { (Fr.) Sacc. }\end{array}$ & 6 & & 6 & & 3 & 4 & & & 7 & 2 & 17 & 2 & & & 9 & & 56 \\
\hline $\begin{array}{l}\text { Fusarium cerealis } \\
\text { (Cooke) Sacc. }\end{array}$ & & & & & 3 & & & & & & & & & & & & 3 \\
\hline $\begin{array}{l}\text { Fusarium } \\
\text { chlamydosporum } \\
\text { Wollenw. \& Reinking }\end{array}$ & 3 & & & & & & & & 1 & & 3 & & & & & & 7 \\
\hline $\begin{array}{l}\text { Fusarium culmorum } \\
\text { (W. G. Sm.) Sacc. }\end{array}$ & 1 & & 1 & & 43 & & & & & & & & 19 & & & & 64 \\
\hline $\begin{array}{l}\text { Fusarium equiseti } \\
\text { (Corda) Sacc. }\end{array}$ & 7 & 52 & 1 & 42 & & 50 & & 62 & 1 & 70 & 70 & 64 & 7 & 42 & 9 & 80 & 557 \\
\hline $\begin{array}{l}\text { Fusarium graminearum } \\
\text { Schwabe }\end{array}$ & & & 3 & & & & & & 5 & & & & & & 2 & & 10 \\
\hline $\begin{array}{l}\text { Fusarium oxysporum } \\
\text { Schltdl. }\end{array}$ & 20 & & 3 & & 2 & & & & 24 & & & & 9 & & 6 & & 64 \\
\hline $\begin{array}{l}\text { Fusarium poae (Peck) } \\
\text { Wollenw. }\end{array}$ & & & 20 & & 3 & & 41 & & 13 & & 9 & 2 & 2 & 2 & 44 & & 136 \\
\hline $\begin{array}{l}\text { Fusarium semitectum } \\
\text { Berk. \& Ravenel }\end{array}$ & & & & & & & 1 & & & & & & & & & & 1 \\
\hline $\begin{array}{l}\text { Fusarium solani } \\
\text { (Mart.) Sacc. }\end{array}$ & & & & & 1 & & & & & & & & & & & & 1 \\
\hline $\begin{array}{l}\text { Fusarium } \\
\text { sporotrichioides Sherb. }\end{array}$ & & & & 4 & & 8 & & & & & & & & & & 14 & 26 \\
\hline
\end{tabular}


cd. table 4

\begin{tabular}{|c|c|c|c|c|c|c|c|c|c|c|c|c|c|c|c|c|c|}
\hline $\begin{array}{l}\text { Fusarium tricinctum } \\
\text { (Corda) Sacc. }\end{array}$ & 5 & & 2 & & 2 & & & & 1 & 2 & & 2 & & & 1 & 2 & 17 \\
\hline Fusarium spp. & 1 & & 4 & & 3 & 2 & & & 4 & & & & & & 1 & & 15 \\
\hline $\begin{array}{l}\text { Gelasinospora reti- } \\
\text { culispora (Greis et } \\
\text { Greis Dengler) C.et M. }\end{array}$ & & & & & & & & & & & & & & & & & \\
\hline Moreau & & & & & & & & & 3 & & & & & & & & 3 \\
\hline $\begin{array}{l}\text { Gliocladium } \\
\text { penicillioides Corda }\end{array}$ & & & & 2 & & & & & & & & & & & & & 2 \\
\hline $\begin{array}{l}\text { Gliomastix murrorum } \\
\text { (Corda) S. Hughes }\end{array}$ & 1 & & & 2 & & & & & & & & & & & & & 3 \\
\hline $\begin{array}{l}\text { Gonatobotrys simplex } \\
\text { Corda }\end{array}$ & & & & & & & & & 1 & & & & & & & & 1 \\
\hline $\begin{array}{l}\text { Humicola fuscoatra } \\
\text { Traaen }\end{array}$ & & & & 2 & & & & & & & & & & & & & 2 \\
\hline $\begin{array}{l}\text { Humicola grisea } \\
\text { Traaen }\end{array}$ & & & & & & & & & 4 & & & & & & & & 4 \\
\hline $\begin{array}{l}\text { Microdochium nivale } \\
\text { (Fr.) Samuels \& I. C. } \\
\text { Hallett }\end{array}$ & 5 & & 1 & & & & 1 & & 2 & & & & 1 & & & & 10 \\
\hline $\begin{array}{l}\text { Monodyctis glauca } \\
\text { (Cooke et Harkn.) } \\
\text { Hughes. }\end{array}$ & & & 3 & & & & & & 4 & & 1 & & & & 1 & & 9 \\
\hline $\begin{array}{l}\text { Monodyctis levis } \\
\text { (Wiltsh.) Hughes. }\end{array}$ & & & & & & & & & 3 & & & & & & & & 3 \\
\hline $\begin{array}{l}\text { Mucor circinelloides } \\
\text { (Cooke et Harkn.) }\end{array}$ & & & & & & & & & & & & & & & & & \\
\hline Mucor hiemalis & & & & & & & & & & & & & & & & & \\
\hline Wehmer & & 16 & & 22 & & 16 & & 28 & 3 & 14 & & 26 & 2 & 30 & 1 & 8 & 166 \\
\hline $\begin{array}{l}\text { Mucor racemosus } \\
\text { Fresen. }\end{array}$ & & & & & & & 1 & & & & & & & & & & 1 \\
\hline $\begin{array}{l}\text { Periconia macrospino- } \\
\text { sa Lefebvre \& Aar. } \\
\text { G. Johnson }\end{array}$ & & & & & & & & & & & 4 & & & & & & 4 \\
\hline $\begin{array}{l}\text { Rhizopus nigricans } \\
\text { Ehrenb. }\end{array}$ & & & & & & & & 2 & & & & & & & & & 2 \\
\hline $\begin{array}{l}\text { Scopulariopsis brevicu } \\
\text { alis (Sacc.) Bainier }\end{array}$ & & & & & & & & & & & & 2 & & & & & 2 \\
\hline $\begin{array}{l}\text { Scopulariopsis } \\
\text { brumptii Salv.-Duval }\end{array}$ & & & & 2 & & 2 & & & & & & & & & & & 4 \\
\hline $\begin{array}{l}\text { Trichoderma hamatum } \\
\text { (Bonord.) Bainier }\end{array}$ & & & & & & 2 & & & & & & & & & & & 2 \\
\hline $\begin{array}{l}\text { Trichoderma polyspo- } \\
\text { rum (Link) Rifai }\end{array}$ & & & 1 & & & & & & & & & & & & & & 1 \\
\hline $\begin{array}{l}\text { Trichoderma viride } \\
\text { Schumach. }\end{array}$ & & & 1 & & & & & & & & & & & & & & 1 \\
\hline non sporulating fungi & & & & & & 2 & & & & & & 2 & & & 1 & & 5 \\
\hline number of isolates & 182 & 160 & 216 & 214 & 235 & 196 & 211 & 214 & 208 & 196 & 198 & 222 & 209 & 184 & 195 & 220 & \\
\hline $\begin{array}{l}\text { number of isolates } \\
\text { from trials }\end{array}$ & & & & & & & & & 4 & & & & & & 4 & & 3260 \\
\hline
\end{tabular}


Table 5

Fungi isolated from grains of winter barley, variety Sezam, 5 months after harvest.

\begin{tabular}{|c|c|c|c|c|c|c|c|c|c|c|c|c|c|c|c|c|c|}
\hline \multirow{3}{*}{ Fungal species } & \multicolumn{17}{|c|}{5 month after harvest } \\
\hline & \multicolumn{2}{|c|}{ A } & \multicolumn{2}{|c|}{ B } & \multicolumn{2}{|c|}{$\mathrm{C}$} & \multicolumn{2}{|c|}{$\mathrm{D}$} & \multicolumn{2}{|c|}{ E } & \multicolumn{2}{|c|}{$\mathrm{F}$} & \multicolumn{2}{|c|}{ G } & \multicolumn{2}{|c|}{$\mathrm{H}$} & \multirow{2}{*}{$\begin{array}{l}\text { Suma } \\
\text { Total }\end{array}$} \\
\hline & 2004 & 2005 & 2004 & 2005 & 2004 & 2005 & 2004 & 2005 & 2004 & 2005 & 2004 & 2005 & 2004 & 2005 & 2004 & 2005 & \\
\hline Acremoniella atra (Corda) & & & & & & & & & & & & & & & & & \\
\hline Sacc. & 1 & & 1 & & & & & & & & & & & & & & 2 \\
\hline Alternaria alternata (Fr.) & & & & & & & & & & & & & & & & & \\
\hline Keissl. & 81 & 117 & 73 & 135 & 88 & 116 & 88 & 92 & 70 & & 59 & 59 & 40 & & 81 & 91 & 1190 \\
\hline Arthrinium & & & & & & & & & & & & & & & & & \\
\hline sphaerospermum Fuckel & & & & & & & & & & & & 1 & & & & & 1 \\
\hline $\begin{array}{l}\text { Aureobasidium bolleyi } \\
\text { (R. Sprague) Arx }\end{array}$ & & & & & 1 & & & & 6 & & & & 1 & & 2 & & 10 \\
\hline $\begin{array}{l}\text { Bipolaris sorokiniana } \\
\text { (Sacc.) Shoemaker }\end{array}$ & 14 & 2 & 13 & 2 & 7 & 2 & 8 & 4 & 11 & 3 & 9 & 1 & 7 & 3 & 4 & 17 & 107 \\
\hline Botrytis cinerea Pers. & & 1 & & & & & & 4 & & 1 & & 5 & & 2 & & & 13 \\
\hline $\begin{array}{l}\text { Cylindrocarpon destructans } \\
\text { (Zinssm.) Scholten }\end{array}$ & 6 & & 2 & & 22 & & 10 & & 12 & & 12 & & 14 & & 10 & & 88 \\
\hline Epicoccum purpurascens & & & & & & & & & & & & & & & & & \\
\hline Ehrenb. & 26 & 21 & 80 & 20 & 20 & 38 & 19 & 33 & 24 & 32 & 35 & 45 & 30 & 30 & 17 & 40 & 510 \\
\hline $\begin{array}{l}\text { Fusarium avenaceum } \\
\text { (Fr.) Sacc. }\end{array}$ & 14 & & 16 & & 31 & & 67 & & 28 & 3 & 55 & 16 & 22 & & 57 & 1 & 310 \\
\hline $\begin{array}{l}\text { Fusarium cerealis } \\
\text { (Cooke) Sacc. }\end{array}$ & & & 4 & & & & & & & & 5 & & & & & & 9 \\
\hline $\begin{array}{l}\text { Fusarium chlamydosporum } \\
\text { Wollenw. \& Reinking }\end{array}$ & & & 2 & & & & & & & & & & & & & & 2 \\
\hline $\begin{array}{l}\text { Fusarium concolor } \\
\text { Reinking }\end{array}$ & & & & & & & & & & 4 & & & & & & & 4 \\
\hline $\begin{array}{l}\text { Fusarium culmorum } \\
\text { (W. G. Sm.) Sacc. }\end{array}$ & & 5 & 9 & 7 & 1 & 21 & & 29 & & 24 & & 5 & & 22 & 1 & 4 & 128 \\
\hline $\begin{array}{l}\text { Fusarium equiseti } \\
\text { (Corda) Sacc. }\end{array}$ & 18 & 45 & 5 & 23 & 13 & 15 & 3 & 23 & 2 & 126 & 4 & 65 & 6 & 137 & 7 & 41 & 533 \\
\hline $\begin{array}{l}\text { Fusarium fusarioides } \\
\text { (Gonz. Frag. \& Cif.) }\end{array}$ & & & & & & & & & & & & & & & & & \\
\hline C. Booth & & 1 & & & & & & 4 & & 1 & & 12 & & 2 & & & 20 \\
\hline $\begin{array}{l}\text { Fusarium graminearum } \\
\text { Schwabe }\end{array}$ & & & 1 & & & & & & & & & & 2 & & & & 3 \\
\hline $\begin{array}{l}\text { Fusarium oxysporum } \\
\text { Schltdl. }\end{array}$ & 43 & 3 & 56 & 6 & 4 & 8 & 1 & 10 & 5 & 8 & & 9 & 11 & 4 & 6 & 6 & 180 \\
\hline $\begin{array}{l}\text { Fusarium poae } \\
\text { (Peck) Wollenw. }\end{array}$ & & & & 1 & & & & & & & & 5 & & 1 & & 1 & 8 \\
\hline $\begin{array}{l}\text { Fusarium semitectum } \\
\text { Berk. \& Ravenel }\end{array}$ & & & & 1 & & & & & & & & & & & & & 1 \\
\hline $\begin{array}{l}\text { Fusarium sporotrichioides } \\
\text { Sherb. }\end{array}$ & & & & & & & & & 2 & & & & & & & & 2 \\
\hline Fusarium spp. & 2 & & 1 & & & & 3 & & & & 1 & 3 & & & & & 10 \\
\hline $\begin{array}{l}\text { Fusarium tricinctum } \\
\text { (Corda) Sacc. }\end{array}$ & 1 & & 5 & & 2 & & 1 & & 7 & & 1 & & & & 1 & 1 & 19 \\
\hline $\begin{array}{l}\text { Gliocladium virens } \\
\text { J. H. Mill., Giddens }\end{array}$ & & & & & & & & & & & & & & & & & \\
\hline \& A. A. Foster & & & & & & & & & & & & 1 & & & & & 1 \\
\hline
\end{tabular}


cd. table 5

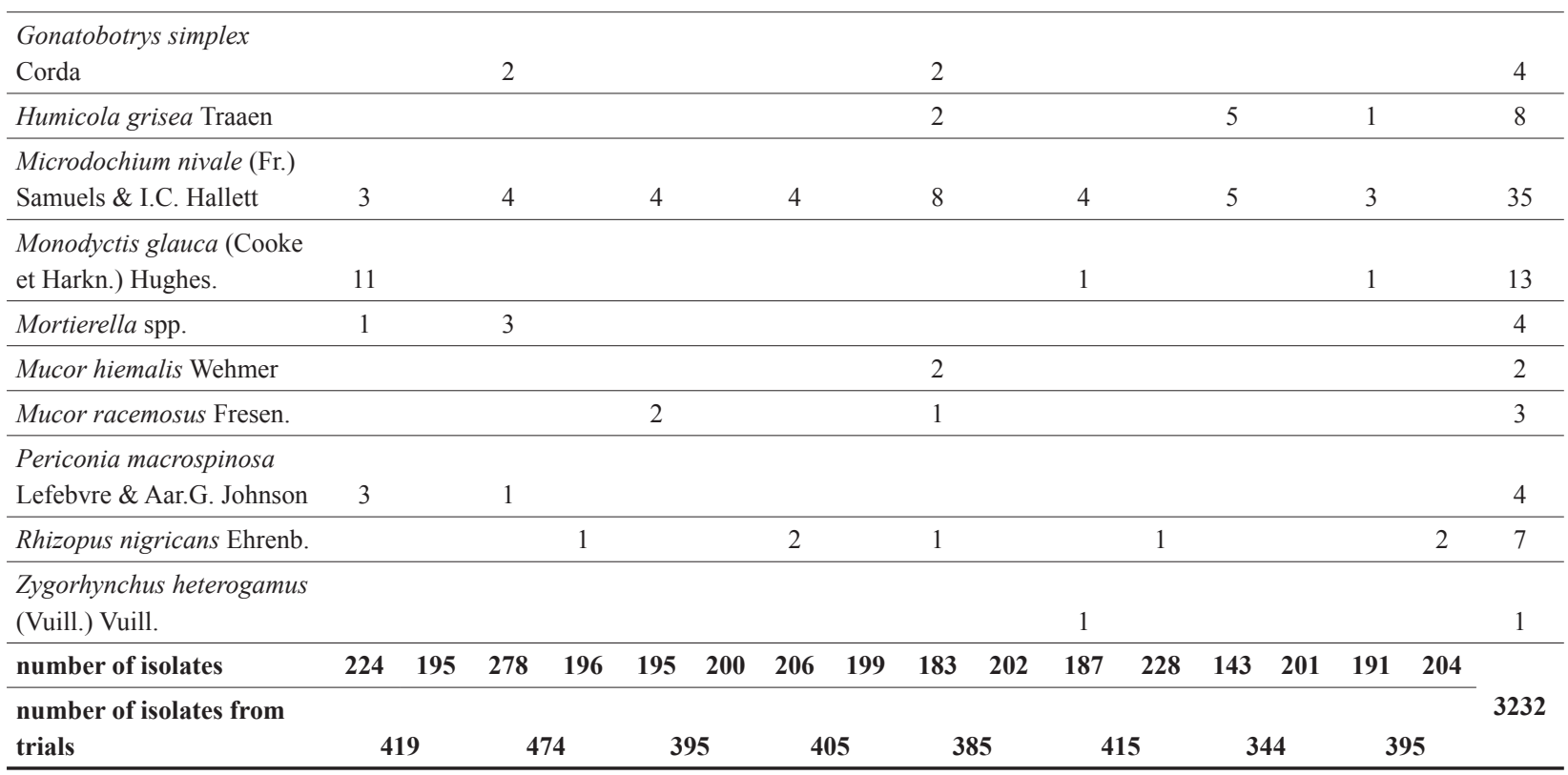

\section{DISCUSSION}

The investigated grain of malting barley, var. Sezam and Prestige, was colonised after the harvest by typical "field" fungi: Alternaria alternata, Epicoccum purpurascens and representatives of the genus Fusarium. In literature, we find reports on the common occurrence of these fungi on cereal grains and seeds of many plants ( $\mathrm{N}$ a r k i e w i c z - J o d k o, 1986; M a j c hrzak, 1998; P arry et al. 1995). In own study, the species Alternaria alternata was the most frequently isolated fungus, accounting for from $36 \%$ up to $45 \%$ of total isolates. Alternaria alternata is a facultative parasite which synthesises toxic metabolites: alternariol $(\mathrm{AOH})$, alternariol methyl ether (AME), altenuene (ALT), altertoxin I, II and III (ATX-I, - II, and - III), tenuazonic acid (TeA) ( $\mathrm{L}$ i et al. 2001; S c o t $t$ 2001). Toxic metabolites produced by this species may inhibit the growth of cereal shoots, as well as they exhibit a toxic effect on cells of mammals ( $\mathrm{N}$ a r k i e w i c Z - J o d ko, 1986; Silvana da Mottia and Soares, 2000; Wat a nabe et al. 2007). The harmfulness of fungal toxins from the genus Alternaria is related to their very common occurrence in the environment, primarily on affected grains and fruit (Wa t a n a be et al. 2007). As reported by Brazaus ki e n è, storage of grain at a temperature of $25^{\circ} \mathrm{C}$ for a period of 6-8 months reduces seed infection by Alternaria spp. by $50 \%$ (B r a z a u s k i e n è et al. 2006). But the lowering of storage temperature does not reduce the level of infection by fungi belonging to this genus, since they are tolerant to low temperatures $(\mathrm{M} \mathrm{a} \mathrm{j} \mathrm{c} \mathrm{hr} \mathrm{z} \mathrm{a} \mathrm{k,}$
1998). Therefore, seed infection by fungi of the genus Alternaria can be of great significance in regions characterised by a cool climate ( $\mathrm{S} \mathrm{m}$ it h et al. 1988).

In our study, fungi of the genus Fusarium were also isolated in a great number. They belong to widely distributed plant pathogens inducing many plant diseases and producing a number of phytotoxic compounds (B row n et al. 2006; N i e s s e n, 2007). Fusarium equiseti was the species which occurred most numerously on malting barley grains. As reported by A ig be and F aw ole (1999), this fungus occurs most frequently in regions characterised by a warm climate, though its presence has been observed on grains of cereals grown in the former USSR countries as well as in Norway and France ( $\mathrm{M}$ a r a s a s et al. 1987, M orrison et al. 2002, I o o s et al. 2004). In Poland the sporadic occurrence of this species on barley grains has been described by $\mathrm{P}$ e rk ow s k i et al. (1997). According to many researchers, this fungus is not included in the Fusarium complex inducing ear fusariosis (L o g r i e c o et al. 2003), but it may cause root diseases, decay of tubers, fruit and seeds of different plants, including cereals ( $\mathrm{A}$ ig be and $\mathrm{Faw}$ ole 1999; Demirci and Dane 2003; Fedel-Moen and $\mathrm{H}$ arris, 1987). F. equiseti synthesises numerous secondary metabolites of different toxicity, among others, trichotecens $\mathrm{A}$ and $\mathrm{B}$, butenolide, equisetin, zearalenon and fusarochromanone ( $\mathrm{M}$ or ris o $\mathrm{n}$ et al. 2002).

In our study, the greatest number of fungi of the genus Fusarium was found after the application of $1 / 2$ of a dose of Amistar (azoxystrobin) used at the stage 
$\mathrm{BBCH}$ 42. M a g a n et al. (2002) have found that low doses of azoxystrobin may stimulate the production of mycotoxins both in in vitro conditions and on wheat grains.

Other authors have reported that the application of triadimenol doses sublethal to Fusarium graminearum resulted in increased production of DON and zearalennon ( $\mathrm{R}$ a m i r e $\mathrm{z}$ et al. 2004). In the opinion of R a m ire z et al. (2004), the application of chemical treatment partially reduces the incidence of typically toxinogenic fungi.

Among potentially pathogenic species, Bipolaris sorokiniana, Botrytis cinerea and Cylindrocarpon destructans were isolated. They are known polyphags affecting many plants. According to $\mathrm{Eac}$ i c o w a et al. (1990, 1992), barley is susceptible to infection by Bipolaris sorokiniana at each growth stage. Grain yield losses as a result of damage caused by this pathogen may reach $30 \%$. The occurrence of Botrytis cinerea, a fungus which is rarely mentioned as a cereal pathogen, on barley is also worth noting. However, in the opinion of the same authors, this species, as an occasional pathogen, is sometimes one of the causes of stem base diseases of barley ( $\mathrm{K}$ a c i c o w a et al. 1990, 1992). According to Michalski and Horoszkiewicz (2003), many pathogens are transferred with plant seeds.

\section{CONCLUSIONS}

1. Grains of both studied varieties of malting barley were colonised by typical field fungi, such as: Alternaria alternata, Epicoccum purpurascens, and fungi of the genus Fusarium.

2. Among fungi potentially pathogenic to cereals, Fusarium equiseti, F. avenaceum, F. oxysporum and Bipolaris sorokiniana were isolated in the greatest number.

3. Grains of malting barley var. Prestige were colonised by fungi more numerously.

4. After the 5-month storage period, more pathogenic fungi, mainly of the genus Fusarium, were isolated from barley grains. Among these fungi, the species $F$. equiseti accounted for the largest percentage share on stored grains.

5. The largest number of fungi of the genus Fusari$u m$ was isolated from both malting barley varieties from the trial objects on which $1 / 2$ of azoxystrobin dose was applied (trial object F).

6. Fungi of the genus Fusarium occurred least frequently on malting barley grains in the combination $\mathrm{G}$ (Dubelt + Corbel + Amistar + Artea).

7. The application of the fungicide Amistar (azoxystrobin) reduced most strongly the occurrence of the species Alternaria alternate; this species occurred on grains from the plants obtained from the combination B (Dubelt J).

\section{REFERENCES}

Aigbe S. O., Fawole B. 1999. A cowpea seed rot disease caused by Fusarium equiseti identified in Nigeria. Plant Disease. 83: 964.

Brazauskienė I., Petraitienė E., Mankevičienė A. 2006. Effects of genotype and environmental factors on rape seed contamination with mycotoxins and mycotoxin-producing fungi. Ekologija, 3. P: 14-20.

Brown D. W., Butchko R. A. E., Proctor R. H. 2006. Fusarium genomic resources: Tools to limit crop diseases and mycotoxin contamination. Mycopath. 162:191-199.

Christensen C. M., 1972. Micoflora and seed detoration. In Roberts E.M. (ed.) Viability of Seeds. Chapman and Hall, LTD, London, 59-93.

Demirci E., Dane E. 2003. Identification and pathogenicity of Fusarium spp. from stem bases of winter wheat in Erzurum, Turkey. Phytoparasitica. 31 (2): 170-173.

Fedel-Moen R., Harris J. R. 1987. Stratified distribution of Fusarium and Bipolaris on wheat and barley with dryland root rot in South Australia. Plant Pathol. 36: 447-454.

Ioos R., Belhadj A., Menez M. 2004. Occurrence and distribution of Microdochium nivale and Fusarium species isolated from barley, durum and soft wheat grains in France from 2000 to 2002. Mycopathol. 158: 351-362.

Knudsen I. M. B., Hocken hul1 J., Jensen D. F. 1995. Biocontrol of seedling diseases of barley and wheat caused by Fusarium culmorum and Bipolaris sorokiniana: effects of selected fungal antagonists on growth and yield components. Plant Pathol. 44 (3): 467-477.

Łacicowa B., Kiecana I., Pięta D. 1990. Choroby podsuszkowe jęczmienia jarego (Hordeum sativum L.) uprawianego w Lubelskiem. / Stem base diseases of spring barley (Hordeum sativum L.) grown in the Lubelskie Voivodship (region). Rocz. Nauk Roln. Seria E, $20(1 / 2): 7-15$.

Łacicowa B., Kiecana I., Pięta D. 1992. Choroby podsuszkowe jęczmienia jarego (Hordeum sativum L.) uprawianego na czarnoziemach. / Stem base diseases of spring barley (Hordeum sativum L.) grown on chernozem soils. Materiały XXXII Sesji Naukowej IOR, Poznań 1992: 66-70.

Li F. Q., Toyazaki N., Yoshizawa T. 2001. Production of Alternaria mycotoxins by Alternaria alternate isolated from weather-damaged wheat. J. Food Protect. 64: 567571.

Logrieco A., Bottalico A., Mule G., Moretti A., Perrone G. 2003. Epidemiology of toxigenic fungi and their associated mycotoxins for some Mediterranean crops. Europ. J. Plant Pathol. 109 (7): 645-667.

Magan N., Hope R., Colleate A, Baxter E. S. 2002. Relationship between growth and mycotoxin production by Fusarium species, biocides and environment. Europ. J. Plant Pathol. 108: 685-690. 
Majchrzak B., 1998. Wpływ stresu chłodno-wodnego na kiełkowanie nasion i zdrowotność siewek wybranych roślin strączkowych. / The effect of cold water stress on seed germination and seedling health of selected leguminous plants. Rozprawy i monografie 1:1-56 Acta Academiae Agriculture ac Technicae Olstenensis, Olsztyn.

Marasas W. F. O., Nelson P. E., Tousson T. A. 1984. Toxigenic Fusarium species, Identity and Mycotoxicology. University Park: Pennsylvania State University Press: 328.

Mathre D. E. 1997. Compendium of Barley Disease, 2nd Ed. APS Press, St. Paul.

Michalski T., Horoszkiewicz J. 2003. Grzyby zasiedlające ziarno owsa nagiego i oplewionego w zależności od sposobu ochrony roślin w okresie wegetacji. / Fungi colonising grains of naked and husked oat depending on the plant protection method in the growing period. Biul. IHAR. 209: 211-219.

Morrison E., Thomas Rundberget T., Kosiak B., Aastveit A. H., Bernhoft A. 2002. Cytotoxicity of trichothecenes and fusarochromanone produced by Fusarium equiseti strains isolated from Norwegian cereals. Mycopathol. 153: 49-56.

Morrison E., Thomas Rundberget T., Kosiak B., Aastveit A. H., Bernhoft A. 2002. Cytotoxicity of trichothecenes and fusarochromanone produced by Fusarium equiseti strains isolated from Norwegian cereals. Mycopathol. 153: 49-56.

Morrison E., Thomas Rundberget T., Kosiak B., Aastveit A. H., Bernhoft A. 2002. Cytotoxicity of trichothecenes and fusarochromanone produced by Fusarium equiseti strains isolated from Norwegian cereals. Mycopathol. 153: 49-56.

Munkvold, G., 2003. Mycotoxins in corn: occurrence, impact and management. In: White, P. and Johson, L. (eds.), Corn: Chemistry and Technology (AACC Inc.): 5-71.

Narkiewicz-Jodko M. 1986. Wartość siewna przechowywanego ziarna trzech zbóż w aspekcie fitopatologicznym. / Sowing value of stored grains of three cereals in the phytopathological aspect. Zesz. Nauk. ART, Wrocław, Rozprawy, 55.

Narkiewicz-Jodko M. 1991. Wpływ warunków zbioru na mikroflorę przechowywanego ziarna pszenżyta ozimego. / The effect of harvest conditions on the microflora of stored grains of winter triticale. Biul. IHAR 180: 33-41.

Parry D. W., Jenkinson P., Mcleod L. 1995. Fusarium ear blight (scab) in small-grain cereals - a review. Plant Pathol. 44 (2): 207-238.

Perkowski J., Jeleleń H., Kiecana I., Goliński P. 1997. Natural contamination of spring barley with group A trichothecene mycotoxins in southeastern Poland. Food Additives and Contaminants. 14: 321-325.
Pięta D. 1997. Wybrane zagadnienia z fitopatologii. / Selected issues of phytopathology. Wyd. AR Lublin, 84.

Ramirez M. L., Chulze So. A, Magan N. 2004. Impact of environmental factors and fungicides on growth and deoxinivalenol production by Fusarium graminearum isolates from Argentinian wheat. Crop Protection. 23: 117-125.

Šarić M., Škrinjar M., Dimić G., Filipović N., Rašić J. 1997. Changes in hygienic and technological wheat quality caused by mould infection. Acta Alimentaria. 26: 255-269.

Scott P. M. 2001. Analysis of agricultural commodities and foods for Alternaria mycotoxins. Journal of AOAC International. 84: 1809-1817.

Silvana Da Mottia, Lucia M. Valente Skares. 2000. A method for the determination of two Alternaria toxins, Alternariol and Alternariol monomethyl ether, in tomato products. Braz. J. Microbiol. 31. P: 315-320.

Smith I. M., Dunez J., Phillips D. H., Lelliott R. A., Archer S. A. 1988. European Handbook of Plant Diseases. Oxford, London: Blackwell Sci. Pub Aigbe i Fawole 1999.

Watanabe I., Kakishima M., Adachi Y., Nakajima H. 2007. Potential mycotoxin productivity of Alternaria alternata isola.

\section{Stan zdrowotny ziarna jęczmienia browarnego w zależności od sposobu ochrony przed chorobami}

\section{Streszczenie}

Badania polowe prowadzono w latach 20042005. Analizę mikologiczną nasion jęczmienia browarnego Prestige i Sezam wykonywano 2 krotnie: na ziarnie przechowywanym przez $30 \mathrm{dni}$ oraz na ziarnie przechowywanym przez okres pięciu miesięcy. W badaniach stwierdzono wpływ ochrony fungicydowej na skład gatunkowy i liczebność grzybów zasiedlających ziarno obu odmian jęczmienia browarnego. Ziarno obydwu odmian jęczmienia browarnego zasiedlone było przede wszystkim przez gatunki grzybów polowych: Alternaria alternata, Epicoccum purpurascens oraz grzyby z rodzaju Fusarium. Wydłużenie okresu przechowywania ziarna do 5 miesięcy spowodowało wzrost udziału gatunków patogenicznych. 